\title{
Coping With COVID-19: Pandemic Life and Problematic Sexual Behaviour
}

\author{
Heather Moulden, ${ }^{1,2}$ John Bradford ${ }^{1-3}$ \\ ${ }^{1}$ Forensic Psychiatry Program, St. Joseph's Healthcare Hamilton, Hamilton, Canada; ${ }^{2}$ Department of Psychiatry and \\ Behavioural Neuroscience, McMaster University, Hamilton, Canada; ${ }^{3}$ Department of Psychiatry, University of Ottawa, \\ Ottawa, Canada
}

As a result of COVID-19 related restrictions around the globe, individuals have experienced a stark shift in the way we socialize and connect. This has impacted many facets of people's lives, one being sexual experience and expression. Although the fact that sex and sexuality were affected by the pandemic and the public health measures and restrictions is no surprise, the specific impacts are proving to be quite fascinating and unexpected. On the one hand, we may predict increased intimacy among partners due to closer proximity and more time together. However, a counter point could be that all that time together combined with the stress of the pandemic suffocated desire. And what about sexual interests? How and why might those be a casualty of pandemic life?

Recent research has examined how lockdowns affect sexuality. For example, in a large online survey of 1,559 adults from predominantly western countries, respondents were asked about the pandemic's impact on their sex life [1]. The findings confirmed that life during the pandemic was related to reduced desire, and both partnered and solo sexual activity. However, among a small group of young, single people, the authors described increased sexual activity and diversity compared with pre-COVID sexual interests and activities, such that their interests became broader after COVID-19 started. In fact, 20\% of participants reported that they had tried a new sexual behaviour during the pandemic [1]. Interestingly, this group also described feeling more stressed and lonelier, which raises questions about the use of sex as a coping mechanism for psychological distress.

This issue of sex and coping was explored further using a web-based survey that inquired about the sexual functioning of approximately 650 adult men and women during COVID-19 confinement in Portugal. Interestingly, psychological adjustment mediated the relationship between confinement and lower sexual functioning (e.g., satisfaction, erectile dysfunction) for men, but not women [2], suggesting that a person's stress and capacity to cope with stress can impact one's sexual expression, functioning, and satisfaction. Another recent study provided further evidence of the increased reliance on sex as coping during the pandemic [3]. This online survey in the U.K. inquired about the effects of social distancing, loneliness, difficulties in emotion regulation, and self-regulation on participants' self-reported coping using sex during lockdown. Based on 789 respondents, results indicated there was no overall increase in coping using sex during lockdown compared with before lockdown. However, upon closer inspection, a third of the sample (30\%) reported increased coping using sex during lockdown compared with before, $29 \%$ reported decreased coping using sex compared to pre-COVID lockdown, and $41 \%$ reported no change in sexual coping related to the pandemic. Among those 
with increased sexualized coping, the predictors included being younger, being male, and having greater emotion dysregulation.

If people are feeling less connected or lonely, and turning to novel or diverse sexual activity to cope, what does this mean for atypical or paraphilic interests? Are we seeing more problematic sexual fantasy or behaviour? These become important questions given the extant literature on individuals who engage in problematic sexual behaviour and sex as coping. This research shows emotion-focused coping, and sexualized coping specifically, among those who sexually abuse children $[4,5]$. Therefore, given emergent findings on changes in atypical sexual behaviour related to the pandemic, we must question the role that coping may play. Although no research on this has been published yet, a recent paper reported increased website activity for online treatment for the use of child sexual abuse material (CSAM) [6]. Not only did the authors find a four-fold increase in the number of users pre- to post-lockdown, but they also observed how chat themes changed with the pandemic. Specifically, users evidenced an increased preoccupation with child sexual abuse and seeking out more extreme CSAM on the darknet, and self-reported relapses among those who had previously quit accessing CSAM. Other concerning themes included reported increased access to children due to home-schooling or babysitting, with instructions about how to access children to produce and share more CSAM.

While reports of sexual abuse rates during the pandemic are not available yet, research into other types of violence related to confinement and other public health measures, suggests that official reporting decreased [7]. However, this finding may more accurately reflect the increased barriers to reporting, such as restricted access to community and support services, as well as personal supports, and confidants; loss of opportunity for private disclosure to teachers or other trusted adults; and proximal or physical interference by the individual perpetrating violence.
As we continue to live through this pandemic, the ways in which it affects relationships and sex (including how we adapt to these changes) presents many opportunities for learning. Of particular interest is the effects of social isolation on sexuality and expression, and when this becomes problematic. So far, it seems that how we cope with the pandemic (and all its impacts) mediates the ultimate effect. However, those who may have vulnerabilities to atypical or problematic sexual interests and behaviours may be at particular risk given the potentiating effects of stress and loss in a time of poor access to supports and services. The emerging research is also beginning to reveal the indirect costs of the pandemic to sexuality, including effects on marital and partnered relationships, sexual functioning, problematic sexual interests, and sexual abuse, which for now appear to be buried, even more than before. It appears that COVID-19 has had many deleterious impacts on sexuality, the true extent of which we may only fully know in the years to come.

While we work to better understand how the COVID-19 pandemic changed sex, we believe that clinical research and practice should attend to both sexual functioning and coping in general, but with particular attention to those with limited social and practical resources, those with psychological vulnerabilities to maladaptive coping (e.g., substance misuse), and those with histories of problematic sexual interests and behaviours. Furthermore, we must go beyond simply the broad pandemic experience to better understand the specific risks-such as loss of work, social isolation, health-related anxiety, trauma, loss, and financial strain (to name but a few) - and the liability they confer on sexual behaviour and through what means. This is complex work, the foundation of which is being built as we try to answer these important questions. But more crucially, we must act quickly and correctly to help those in need and protect those at risk.

Conflict of Interest: none 


\section{References}

1. Lehmiller JJ, Garcia JR, Gesselman AN, Mark KP. Less sex, but more sexual diversity: Changes in sexual behavior during the COVID-19 coronavirus pandemic. Leisure Sciences. 2020;43(1-2):295-304. https://doi.org/10.1080/01490400.2020.1774016.

2. Carvalho J, Campos P, Carrito M, et al. The relationship between COVID-19 confinement, psychological adjustment, and sexual functioning, in a sample of Portuguese men and women. The Journal of Sexual Medicine. 2021;18(7):1191-1197.

https://doi.org/10.1016/j.jsxm.2021.04.007.

3. Gillespie SM, Jones A, Uzieblo K, et al. Coping using sex during the coronavirus disease 2019 (COVID-19) outbreak in the United Kingdom. The Journal of Sexual Medicine. 2021;18(1):50-62. https://doi.org/10.1016/j.jsxm.2020.11.002.

4. Cortoni F, Marshall WL. Sex as a coping strategy and its relationship to juvenile sexual history and intimacy in sexual offenders. Sexual Abuse. 2001;13(1):27-43. https://doi.org/10.1177/107906320101300104.
5. Feelgood S, Cortoni F, Thompson A.

Sexual coping, general coping and cognitive distortions in incarcerated rapists and child molesters. Journal of Sexual Aggression. 2005;11(2):157-170. https://doi.org/10.1080/13552600500073657.

6. Parks A, Sparre C, Söderquist E, Arver S, Andersson G, Kaldo V, et al. Illegal online sexual behavior during the COVID-19 pandemic: A call for action based on experiences from the ongoing prevent it research study. Archives of Sexual Behavior. 2020;49:1433-1435. https://doi.org/10.1007/s10508-020-01750-7.

7. Payne JL, Morgan A, Piquero AR. COVID-19 and social distancing measures in Queensland, Australia, are associated with short-term decreases in recorded violent crime. Journal of Experimental Criminology. 2020:1-25. https://doi.org/10.1007/s11292-020-09441-y.

\section{Corresponding author}

Dr. Heather M. Moulden, Clinical Forensic Psychologist, Forensic Psychiatry Program, St. Joseph's Healthcare Hamilton, West 5th Campus, 100 West 5th Street, Hamilton, ON L8N 3K7 Canada - email: hmoulden@stjosham.on.ca. 